\title{
Jumps in current-voltage characteristics in disordered films
}

\author{
Boris L. Altshuler, ${ }^{1,2}$ Vladimir E. Kravtsov, ${ }^{3}$ Igor V. Lerner, ${ }^{4}$ and Igor L. Aleiner ${ }^{1}$ \\ ${ }^{1}$ Physics Department, Columbia University, 538 West 120th Street, New York, N.Y. 10027, USA \\ ${ }^{2}$ NEC-Laboratories America, Inc., 4 Independence Way, Princeton, N.J. 085540, USA \\ ${ }^{3}$ The Abdus Salam International Centre for Theoretical Physics, P.O. Box 586, 34100 Trieste, Italy \\ ${ }^{4}$ School of Physics and Astronomy, University of Birmingham, Birmingham B15 2TT, United Kingdom
}

\begin{abstract}
We argue that giant jumps of current at finite voltages observed in disordered films of $\operatorname{InO}$, TiN and YSi manifest a bistability caused by the overheating of electrons. One of the stable states is overheated and thus low-resistive, while the other, high-resistive state is heated much less by the same voltage. The bistability occurs provided that cooling of electrons is inefficient and the temperature dependence of the equilibrium resistance, $R(T)$ is steep enough. We use experimental $R(T)$ and assume phonon mechanism of the cooling taking into account its strong suppression by disorder. Our description of details of the $I-V$ characteristics does not involve adjustable parameters and turns out to be in a quantitative agreement with the experiments. We propose experiments for more direct checks of this physical picture.
\end{abstract}

PACS numbers: 72.20.Ht, 73.50.Fq, 73.63.-b

Recent experiments on the $I-V$ characteristics of disordered films of $\operatorname{InO}$ [1, 2] and TiN [3, 4] (Similar findings were reported earlier for amorphous YSi films [5]) are quite intriguing. Ohmic resistance $R$ of these films at temperatures $T<1 K$ demonstrates insulating behavior fit by Arrhenius law $\left(k_{B}=1\right)$

$$
R(T)=R_{0} \exp \left[(\Delta / T)^{\gamma}\right], \quad \gamma=1,
$$

with $R_{0}$ and $\Delta \simeq 1 \div 5 K$ being $T$-independent. Though this behavior is quite interesting by itself [], it is the $I-V$ characteristics at low temperatures which turned out to be most unusual. When the voltage $V$ is increased from $V=0$, the current $\mathcal{I}$ first increases gradually, remaining rather small [highly resistive (HR) state]. At a certain voltage $V_{\mathrm{HL}}, \mathcal{I}$ jumps up by several orders of magnitude and a low resistive (LR) state arises. When the voltage is decreased from $V>V_{\mathrm{HL}}$, an inverse jump between the LR and HR states occurs at a voltage $V_{\mathrm{LH}}<V_{\mathrm{HL}}$. These HR-LR switches persist in a wide range of magnetic fields $B \simeq 0 \div 10 T$, with the threshold voltages $V_{\mathrm{LH}(\mathrm{HL})}$ increasing with $B$. Authors of Refs. [4, 7] assumed that the HR state is a new collective state - "superinsulator". Their calculation was vigorously disputed in Ref. [8].

In this Letter we show that these $I-V$ characteristics can be explained without using new concepts. Our phenomenological approach predicts exactly this behavior when $T$-dependence of the resistance is steep as in Eq. (1) and the electron-phonon (e-ph) thermalization is inefficient at low $T$. We assume that (similar assumptions for a system without the switches were made in Ref. [9])

1. The electron-electron (e-e) interaction is strong enough for electrons being mutually thermalized, i.e. one can introduce their temperature $T_{\mathrm{el}}$ although the system is driven out of equilibrium by a finite voltage;

2. The e-ph interaction is weak, so that electrons can be out of equilibrium with phonons (or any other thermal bath) of temperature $T_{\mathrm{ph}}$, i.e. $T_{\mathrm{el}}>T_{\mathrm{ph}}$;
3. $R(T)$-dependence at a finite voltage is the same as in the ohmic regime, but $T_{\mathrm{el}}(V)$ is substituted for $T$.

These assumptions are sufficient to explain qualitatively the experimental $I-V$ characteristics, most carefully investigated in the accompanying Letter $[2]$.

Phenomenological analysis - Temperature $T_{\mathrm{el}}$ acquired by the electron due to a fixed external voltage $V$ is determined by the balance between the Joule heating and the cooling by the phonon bath (electron-electron collisions conserve the energy and do not affect the heat balance): Before specifying the model for the electronphonon (e-ph) coupling we analyze the cooling using model-independent arguments. The heat balance equation for a sample of volume $\mathcal{V}$ and the electron density of states $\nu$ (assumed to be smooth at the Fermi level) is

$$
\begin{aligned}
& \frac{V^{2}}{R\left(T_{\mathrm{el}}\right)}=\frac{\mathcal{E}\left(T_{\mathrm{el}}\right)}{\tau_{\mathrm{e}-\mathrm{ph}}\left(T_{\mathrm{el}}\right)}-\frac{\mathcal{E}\left(T_{\mathrm{ph}}\right)}{\tau_{\mathrm{e}-\mathrm{ph}}\left(T_{\mathrm{ph}}\right)}, \\
& \frac{1}{\tau_{\mathrm{e}-\mathrm{ph}}(T)}=\left(\frac{T}{\hbar}\right)\left(\frac{T}{\Theta}\right)^{\beta-3},
\end{aligned}
$$

where $\mathcal{E}=\pi^{2} \nu \mathcal{V} T^{2} / 6$. The r.h.s of Eq. 2a obeys the following requirements: (i) it vanishes in the thermal equilibrium; (ii) it provides the entropy growth; (iii) at $T_{\mathrm{el}} \gg T_{\mathrm{ph}}$, it ceases to depend on $T_{\mathrm{ph}}$ as under such condition the radiation of the hot phonons by hot electrons dominates. Factor $T / \hbar$ provides the proper dimensionality for the relaxation rate. The second factor in r.h.s. of Eq. 2b describes a suppression of the e-ph coupling at low $T$. As the phonons are gapless, the suppression is a power law. The energy scale $\Theta$ encodes the strength of e-ph coupling. We will specify $\beta$ and $\Theta$ later.

For a steep enough $R(T)$, there is a region on the $V$ $T_{\mathrm{ph}}$ plane (for $T_{\mathrm{ph}}<T_{\mathrm{ph}}^{\mathrm{cr}}$ and $V^{<}<V<V^{>}$) where Eqs. (2) have two stable solutions: a "hot" LH state with relatively high $T_{\mathrm{el}}$ and a "cold" HR state with $T_{\mathrm{el}} \approx T_{\mathrm{ph}}$. 
Such a situation was discussed in Ref. [10] in connection with the many-body localization [11.

To analyze Eqs. (1) - (2) we use dimensionless variables

$$
t_{\mathrm{el}, \mathrm{ph}} \equiv \frac{T_{\mathrm{el}, \mathrm{ph}}}{\Delta}, \quad v \equiv \frac{V}{V_{0}},
$$

and rewrite Eq. 2al in the form

$$
F\left(t_{\mathrm{el}}, t_{\mathrm{ph}}\right)=v ; F(x, y) \equiv\left[\mathrm{e}^{1 / x^{\gamma}}\left(x^{\beta}-y^{\beta}\right)\right]^{1 / 2} .
$$

The new characteristic voltage, $V_{0}$ emerging in Eq. (3), is natural to define through the electric field scale $V_{0} / L$, independent of the system size:

$$
\frac{V_{0}}{L}=\left(\frac{\pi^{2} \nu \Delta^{3}}{6 \sigma_{0} \hbar}\right)^{1 / 2}\left(\frac{\Delta}{\Theta}\right)^{\frac{\beta-3}{2}} ; \quad \sigma_{0}=\frac{L^{2}}{R_{0} \mathcal{V}} .
$$

The first factor in the r.h.s. of Eq. (5) is uniquely determined as a combination of local characteristics of an isolated system, $\nu, \sigma_{0}$ and $\Delta$, with a correct dimensionality. The problem of nonlinear dissipative transport is meaningless without coupling of the electrons with a thermal bath, i.e. when $\Theta \rightarrow \infty$. This is reflected by the second factor in Eq. (5).

At the bistability boundaries $t_{\mathrm{el}}^{\mathrm{h}, \mathrm{c}}$, the derivatives with respect to $t_{\mathrm{el}}$ of both sides of Eq. (4) are equal, i.e:

$$
(\beta / \gamma) t_{\mathrm{el}}^{\gamma}=1-\left(t_{\mathrm{ph}} / t_{\mathrm{el}}\right)^{\beta}
$$

This equation has two positive solutions if $t_{\mathrm{ph}} \leq t_{\mathrm{ph}}^{\mathrm{cr}}(\beta)$. Here, the critical phonon temperature, $t_{\mathrm{ph}}^{\mathrm{cr}}=T_{\mathrm{ph}}^{\mathrm{cr}} / \Delta$ is

$$
t_{\mathrm{ph}}^{\mathrm{cr}}=(1+\beta / \gamma)^{-\frac{\beta+\gamma}{\gamma \beta}}<1 .
$$

The two solutions $t_{\mathrm{el}}^{\mathrm{c}(\mathrm{h})}\left(t_{\mathrm{ph}}\right)$ of Eq. [6), see Fig. 1, define the region of voltages $v^{<}<v<v^{>}$where two states are locally stable, $v^{<,>}=F\left(t_{\mathrm{el}}^{\mathrm{h}, \mathrm{c}}, t_{\mathrm{ph}}\right)$. The "hot" branch $t_{\mathrm{el}}^{\mathrm{h}}$ turns out to only slightly depend on $t_{\mathrm{ph}}$ :

$$
\left(\frac{\gamma}{\beta+\gamma}\right)^{\frac{1}{\gamma}}=t_{\mathrm{el}}^{\mathrm{h}}\left(t_{\mathrm{ph}}^{\mathrm{cr}}\right) \leq t_{\mathrm{el}}^{\mathrm{h}}\left(t_{\mathrm{ph}}\right) \leq t_{\mathrm{el}}^{\mathrm{h}}(0)=\left(\frac{\gamma}{\beta}\right)^{\frac{1}{\gamma}} .
$$

As the result, $v^{<}\left(t_{\mathrm{ph}}\right)$ is almost independent of $T_{p h}$,

$$
\sqrt{\mathrm{e}}\left(\frac{\beta}{\beta+\gamma}\right)^{\frac{\beta+\gamma}{2 \gamma}}\left(\frac{\mathrm{e} \gamma}{\beta}\right)^{\frac{\beta}{2 \gamma}} \leq v^{<}\left(t_{\mathrm{ph}}\right) \leq\left(\frac{\mathrm{e} \gamma}{\beta}\right)^{\frac{\beta}{2 \gamma}}
$$

Contrarily, for $\beta \gg 1$, the "cold" branch may be well approximated (except narrow vicinity of $t_{\mathrm{ph}}^{\mathrm{cr}}$ ) by

$$
t_{\mathrm{el}}^{\mathrm{c}}-t_{\mathrm{ph}} \approx t_{\mathrm{ph}}^{\gamma+1} / \gamma<t_{\mathrm{ph}} / \beta .
$$

For the upper bistability boundary, we find

$$
v^{>}\left(t_{\mathrm{ph}}\right) \approx[\beta /(e \gamma)]^{1 / 2} t_{\mathrm{ph}}^{(\beta+\gamma) / 2} \exp \left[1 /\left(2 t_{\mathrm{ph}}^{\gamma}\right)\right] .
$$

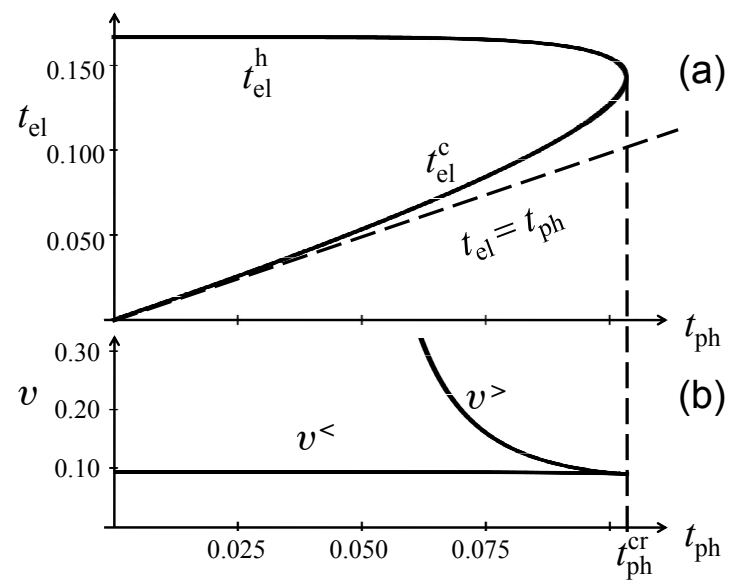

FIG. 1: Dependence of the dimensionless electronic temperature (a) and voltage (b) bistability boundaries on the bath temperature $t_{\mathrm{ph}}$. Here $t_{\mathrm{el}}^{\mathrm{c}}$ and $v^{>}$are the boundaries for the HR ("cold") state and $t_{\mathrm{el}}^{\mathrm{h}}$ and $v^{<}$for the LR ("hot") state. Both plots are for $\beta=6, \gamma=1$.

At each voltage in the bistability interval $v^{<}<v<v^{>}$ one of the states is metastable. As it is usual for the first order phase transition the voltages, where the switches between HR and LR states happen ( $V_{\mathrm{HL}}$ for $\mathrm{HR} \rightarrow \mathrm{LR}$ and $V_{\mathrm{LH}}$ for $\mathrm{LR} \rightarrow \mathrm{HR}$ switches), are determined by kinetics of the decay of metastable states. Theoretical analysis of this decay and evaluation of $V_{\mathrm{HL}, \mathrm{LH}}$ is beyond the scope of this paper. Here we can predict only their bounds

$$
V_{0} v^{<}=V^{<}<V_{\mathrm{LH}}<V_{\mathrm{HL}}<V^{>}=V_{0} v^{>} .
$$

The difference between the local instability and the metastable state decay transition can be ascertained from the slope $d \mathcal{I} / d V$ near the transition, see Fig. 2.

Finally, it is important to emphasize that Eqs. (3) (5) imply a non-trivial scaling of the bistability bounds,

$$
V^{<,>}=\Delta^{\beta / 2} f^{<,>}\left(T_{\mathrm{ph}} / \Delta\right),
$$

which should apply to $V_{\mathrm{HL}}$, LH provided that the switches are close to (a)-type, see Fig. 2 .

The $I-V$ characteristics is most conveniently visualized by calculating the non-linear conductance $G$

$$
\mathcal{I}=G\left(V, T_{\mathrm{ph}}\right) V ; \quad G\left(T_{\mathrm{ph}}, V\right)=\frac{\exp \left[-t_{\mathrm{el}}(v)^{-\gamma}\right]}{R_{0}},
$$

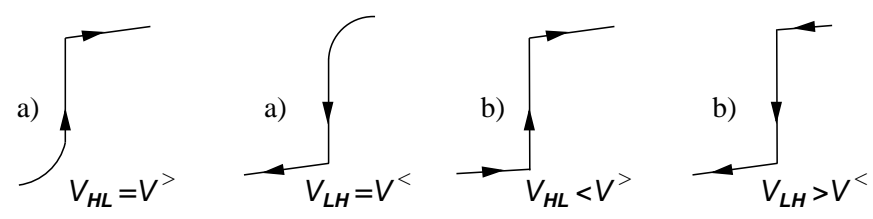

FIG. 2: The schematic $I-V$ characteristics for the switches occuring through the local instability [type (a)] and through the decay of metastable state [type (b)]. 
see Eq. (1), where $t_{\mathrm{el}}(v)$ is found from Eq. (4). The $I-V$ curves obtained by substituting the numerical solution of Eq. (4) into Eq. (11) are plotted on Fig. 3. The HR and LR branches are connected by unstable branches shown by dotted lines. The HR $\rightarrow$ LR ("cold" to "hot" state) and LR $\rightarrow$ HR ("hot" to "cold" state) switches are depicted by vertical dashed lines.

If $T_{\mathrm{ph}}$ is not too close to the critical temperature (7), we can use the fact $t_{\mathrm{el}}-t_{\mathrm{ph}} \ll t_{\mathrm{ph}}$, see Eq. 8c , to obtain an analytic description of $G\left(V, T_{\mathrm{ph}}\right)$ for the HR state:

$$
\left(\frac{v}{v^{>}}\right)^{2}=\frac{\mathrm{e} \ln \left[G R\left(T_{\mathrm{ph}}\right)\right]}{G R\left(T_{\mathrm{ph}}\right)} ; \quad 1 \leq G R\left(T_{\mathrm{ph}}\right) \leq \mathrm{e} .
$$

Note, that the dependence is universal, i.e. it holds for arbitrary values of exponents $\beta, \gamma$.

Microscopic input - To quantify the developed phenomenology we rely on conventional theory of normal disordered metals neither seeking a microscopic explanation for, e.g., the Arrhenius law (1) nor involving physics of the insulator-superconducting transition. The current jumps occur in the insulating regime $T<\Delta$. However, the thermal balance equation is valid also for $T \gtrsim \Delta$ and the e-ph coupling should be a continuous function. Thus, our strategy is to use the theory of the e-ph interaction in dirty metals [12, 13 for $T>\Delta$ and extrapolate to the lower temperatures. The cooling rate is determined by the material mass density, $\rho$, and the transverse sound velocity $c_{\mathrm{s}}$. Disorder is known to suppress the e-ph coupling if the wave length of a thermal phonon exceeds the electron elastic mean free path, $\hbar c_{\mathrm{s}} / T \gg \ell$, (as it does for films of Refs. [1, 2, 3, 4, 5] where $c_{\mathrm{s}} \sim 3 \times 10^{5} \mathrm{~cm} / \mathrm{s}$, $T \lesssim 1 K$ and $\ell \lesssim 10 \mathrm{~nm})$. The result is [13]

$$
\frac{\mathcal{E}(T)}{\tau_{\mathrm{e}-\mathrm{ph}}(T)}=\frac{\alpha^{2} k_{\mathrm{F}} \ell \mathcal{V} n_{\mathrm{el}} T^{6}}{\hbar^{4} \rho c_{\mathrm{s}}^{5}}, \quad \alpha=\frac{2 \pi^{2}}{\sqrt{315}} \approx 1.1
$$

where $n_{\mathrm{el}}$ is the conduction electrons density, and $k_{\mathrm{F}}=$ $\left(3 \pi^{2} n_{\mathrm{el}}\right)^{1 / 3}$ is the Fermi momentum [14, 15]. Comparing Eqs. (13) and 2b we obtain

$$
\beta=6, \quad \Theta=\left(\frac{\pi^{2}}{6 \alpha^{2}} \frac{\hbar^{3} \nu \rho c_{\mathrm{s}}^{5}}{n_{\mathrm{el}} k_{\mathrm{F}} \ell}\right)^{1 / 3} .
$$

Substituting Eq. (14) into Eq. (5), and using, at $T>\Delta$, the Drude formula $\sigma_{0}=\left(e^{2} / \hbar\right)\left(k_{\mathrm{F}} \ell\right)\left(n_{\mathrm{el}} / k_{\mathrm{F}}^{2}\right)$, we find

$$
\frac{e V_{0}}{L}=\alpha \frac{k_{\mathrm{F}} \Delta^{3}}{\left(\rho c_{\mathrm{s}}^{5} \hbar^{3}\right)^{1 / 2}}
$$

Remarkably, the elastic mean free-path $\ell$ - the only quantity, which becomes meaningless in the insulator, does not enter electric field scale $V_{0} / L$. It makes us believe that Eq. 15 derived for the metallic regime $T>\Delta$ may be extended to the insulator at $T<\Delta$.

Qualitative comparison with experiment- Several conclusions of our phenomenological consideration can be compared directly with the experimental results:

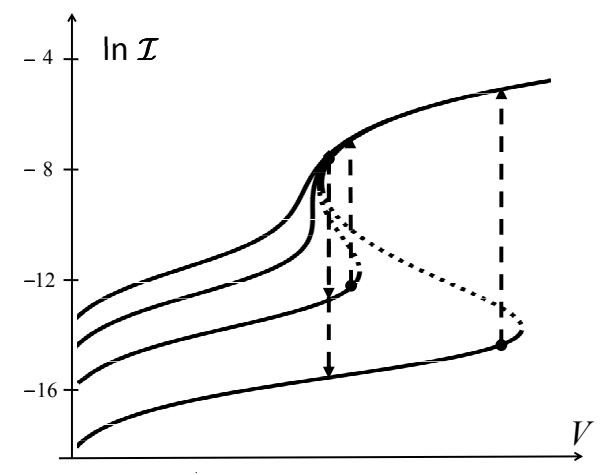

FIG. 3: Universal I-V characteristics for $\beta=6, \gamma=1$ (going from the top) $t_{\mathrm{ph}}=1.15 t_{\mathrm{ph}}^{\mathrm{cr}}$, and $t_{\mathrm{ph}}=t_{\mathrm{ph}}^{\mathrm{cr}}, t_{\mathrm{ph}}=0.90 t_{\mathrm{ph}}^{\mathrm{cr}}$ and $t_{\mathrm{ph}}=0.75 t_{\mathrm{ph}}^{\mathrm{cr}}$. Instable regions on the $\mathrm{S}$-shaped curves are shown by dotted lines which join the upper and lower solid lines at the stability boundaries of the LR ("hot") and HR ("cold") state, respectively. The vertical dashed lines with arrows show possible positions for LR $\rightleftarrows$ HR current jumps. The units of $\mathcal{I}$ and $V$ are arbitrary, but the values of the current switches in the log-scale are universal and in a good agreement with the experimental values.

1. The $I-V$ curves in $[1,2,3,4,5$, look like those on Fig 3 for both $T>T_{\mathrm{ph}}^{\mathrm{cr}}$ and $T<T_{\mathrm{ph}}^{\mathrm{cr}}$. At $T \rightarrow T_{\mathrm{ph}}^{\mathrm{cr}}+0$ the inflection point becomes increasingly pronounced. At $T$ below $T_{\mathrm{ph}}^{\mathrm{cr}}$, hysteretic $\mathrm{HR} \rightarrow \mathrm{LR}$ and $\mathrm{LR} \rightarrow \mathrm{HR}$ current jumps occur. These jumps indeed reach several orders of magnitude;

2. At a rather large interval of voltages the dependence of $\log \mathcal{I}$ on $V$ looks like linear;

3. $V_{L H}$ only slightly changes with $T_{\mathrm{ph}}$, while $V_{\mathrm{HL}}$ substantially increases when phonon temperature is reduced. This is what Eqs. $8 \mathrm{~b}), 8 \mathrm{~d}$ ) predict.

4. Observed $T_{\mathrm{ph}}$-dependence of $V_{\mathrm{HL}}$ is still weaker than the exponential dependence $(8 \mathrm{~d})$. This is consistent, however, with bound (9) and the type (b) HR LR transition (see discussion before Eq. (9), and Fig. 2). Another possible reason for a relatively slow dependence of $V_{H L}$ on $T_{p h}$ is a deviation from the Arrhenius law of Eq. (1).

5. The assumption that $V_{\mathrm{HL}}<V^{>}$and $V_{\mathrm{LH}} \approx V^{<}$implies the shapes of the $I-V$ characteristics to be of type (a) close to LR $\rightarrow$ HR switch and of type (b) in the vicinity of $\mathrm{HR} \rightarrow \mathrm{LR}$ switch (see Fig 2). This is exactly what was observed.

Further analysis of data of Ref. [2] uncovers an intriguing discrepancy: the observed ratio of the nonlinear conductances $G\left(V_{\mathrm{HL}}\right) / G(V \rightarrow 0)$, see Eq. (11), is noticeably bigger than the bounds of Eq. (12) and Fig. 3, valid for any single scale dependence $R(T)$. This discrepancy can be resolved within our approach only by involving an extra temperature scale $T^{*}<T_{\mathrm{ph}}^{\mathrm{cr}}$ in addition to $\Delta$. While the origin of this scale is yet to be understood, the manybody localization [11] is a possibility. 
Quantitative discussion. The magnitude of the LR-HR jump is almost independent of other experimental parameters. Since locations of the jumps between the upper and lower stability boundaries are ill-defined, we can only estimate the order of magnitude. For $T_{\mathrm{ph}}=0.75 T_{\mathrm{ph}}^{\mathrm{cr}}$ the predicted 4-5 orders in magnitude of the current jump (Fig. 3) agrees reasonably with experiment. Experimental cooling is well fitted with $\beta=6$ in Eq. (2), in agreement with the above description of the electron-phonon mechanism. The critical bath temperature can be estimated from the temperature scale $\Delta \approx 1.2 \mathrm{~K}$ : although the Arrhenius law does not give a very good fit, this scale can be rather reliably extracted from the data 2 . Then for $\gamma=1$ and $\beta=6$, we find $T_{\mathrm{ph}}^{\mathrm{cr}} \approx 0.1 \Delta \approx 0.1 \mathrm{~K}$ in agreement with the experimental values 2 .

More quanitative comparison is hindered by the strong sensitivity of the critical temeperature and switching voltages to the choice of $\gamma$, see Eqs. (7) - Eq. (8). For a consistent quantitative comparison with experimental observation, one should fit the experimentally observed equilibrium resistance $R(T)$ into the left-hand-side of the heat balance equation Eq. 2a and solve the resulting equation numerically. This is done in the accompanying experimental Letter 2].

In conclusion, there is a number of strong evidences in favor of the electron overheating being the main cause of the giant $\mathrm{HR} \leftrightarrow \mathrm{LR}$ current jumps observed in 1, 2, 3, 4, 5. Direct detection of the electron overheating through e.g., noise measurements would be an unambiguous proof of this. As the cooling is a rather slow process it looks plausible to perform the time resolved-studies of electron transport, e.g. measure the current caused by a train of voltage pulses as a function of the pulse duty cycle. Such a measurement could also shed some light on the kinetics of the switches. Also, the scaling relation (10) is very characteristic for the overheating mechanism (it can be verified by tuning $\Delta$, e.g., by gentle annealing).

Finally, we emphasize the importance of our interpretation of the data $1,2,3,4,5$, in the context of the general theory of the electron transport. Overheating of the electrons is quite usual in low resistive metals [18. As to insulators (resistance far in excess of $h / e^{2}$ ) the overheating was rarely [9] considered quantitatively, because the conventional mechanisms of the low temperature charge transport are based on phonon-assisted hopping (see, e.g., [19]). We fully realize that our explanation of the current jumps contradicts the standard picture (as well as the Arrhenius law Eq. (1), $\gamma=1$, though). At the same time, we find the arguments in favor for the overheating being observed in Refs. 1, 2, 3, 4, 5, to be quite convincing. If our explanation is confirmed, these experiments should be considered as the first (to the best of our knowledge) reliable evidence of the strong overheating in the insulating state and, thus, of the existence of phonon-independent transport in insulators.

We thank M. Ovadia, B. Sacepe and D. Shahar for sharing with us their experimental results 2] prior to publication and $\mathrm{Yu}$. M. Galperin for reading the manuscript and valuable remarks. V. E. K. is grateful to M. V. Feigel'man for stimulating discussions. V. E. K. and I. V. L. acknowledge kind hospitality extended to them at the Newton Institute (Cambridge). We acknowledge support by EPSRC grant T23725/01, and by the US DOE contract No. DE-AC02-06CH11357.

[1] G. Sambandamurthy, et al, Phys. Rev. Lett. 94, 017003 (2005).

[2] M. Ovadia, B. Sacepe, and D. Shahar, in preparation (2008).

[3] T. I. Baturina, et. al. Phys. Rev. Lett. 99, 257003 (2007).

[4] V. M. Vinokur, et. al., Nature 452, 613 (2008).

[5] F. Ladieu, M. Sanquer, and J. P. Bouchaud, Phys. Rev. B 53, 973 (1996).

[6] D. Kowal and Z. Ovadyahu, Sol. State Commun. 90, 783 (1994); D. Shahar and Z. Ovadyahu, Phys. Rev. B 46, 10917 (1992); V. Gantmakher, et. al. ZhETF 109, 1765 (1996); G. Sambandamurthy, et. al., Phys. Rev. Lett. 92, 107005 (2004).

[7] M. V. Fistul, V. M. Vinokur, and T. I. Baturina, Phys. Rev. Lett. 100, 086805 (2008); arXiv:0806.4311.

[8] K. B. Efetov, M. V. Feigel'man, and P. B. Wiegmann, arXiv:0804.3775 (2008).

[9] M. E. Gershenson et. al., Phys. Rev. Lett. 85, 1718 (2000).

[10] D. M. Basko, I. L. Aleiner, and B. L. Altshuler, Phys. Rev. B 76, 052203 (2007).

[11] D. M. Basko, I. L. Aleiner, and B. L. Altshuler, Ann. Phys. 321, 1126 (2006).

[12] A. Schmid, Z. Phys. 271, 251 (1974).

[13] M. Y. Reizer and A. V. Sergeev, Zh. Eksp. Teor. Fiz. 90, 1056 (1986); V. I. Yudson and V. E. Kravtsov, Phys. Rev. B 67, 155310 (2003).

[14] Note that this phonon relaxation rate is suppressed by a factor of several orders of magnitude in comparison with the usually used formula for clean dense metal, $1 / \tau_{\mathrm{e}-\mathrm{ph}}^{\text {clean-dense }}$. Namely, $1 / \tau_{\mathrm{e}-\mathrm{ph}}=1 / \tau_{\mathrm{e}-\mathrm{ph}}^{\text {clean-dense }} \times$ $\left(n^{*} \ell T / \hbar c_{\mathrm{s}}\right)$, where $n^{*}<1$ is the number of conduction electrons per unit cell. Authors of Ref. [5] estimated the overheating as too small to cause observed voltage switches in YSi films. Effects of overheating (other than bistability) were discussed in Refs. [16, 17]. In Refs. [5, 16] $\tau_{\mathrm{e}-\mathrm{ph}}^{\text {clean-dense }}$ was used which lead to the overestimate of cooling rates by several orders of magnitude.

[15] Equation (13) is obtained within a rather crude jelly model. Even though this does not affect the value of the exponent $\beta=6$, the value of $\Theta$ in Eq. 14 may be off by an unknown numerical factor.

[16] M. V. Golubkov and G. E. Tsydynzhapov, JETP Lett. 71, 516 (2000).

[17] K. A. Parendo, et. al. Phys. Rev. B 74, 134517 (2006).

[18] A. F. Volkov and S. M. Kogan, Sov. Phys. Uspekhi, 11, 881 (1969).

[19] B.I. Shklovskii and A.L. Efros, Electronic Properties of Doped Semiconductors, Springer, Heidelberg (1984). 